Cooperative Effects of an Antifungal Moiety and DMSO on Pore Formation over Lipid Membranes Revealed by Free Energy Calculations

\title{
Kasparyan, Gari
}

2020-10-08

Kasparyan , G, Poojari , C , Rog , T \& Hub , J S 2020 , ' Cooperative Effects of an Antifungal Moiety and DMSO on Pore Formation over Lipid Membranes Revealed by Free Energy Calculations ' , Journal of Physical Chemistry B , vol. 124 , no. 40 , pp. 8811-8821 . https://doi.org/10.1021/a

http://hdl.handle.net/10138/334328

https://doi.org/10.1021/acs.jpcb.0c03359

acceptedVersion

Downloaded from Helda, University of Helsinki institutional repository.

This is an electronic reprint of the original article.

This reprint may differ from the original in pagination and typographic detail.

Please cite the original version. 


\title{
Cooperative effects of an antifungal and DMSO on pore formation over lipid membranes revealed by free energy
}

\section{calculations}

\author{
Gari Kasparyan, ${ }^{\dagger}$, Chetan Poojari, ${ }^{* \dagger,}$ Tomasz Róg, ${ }^{\dagger}$ and Jochen S. Hub*, \\ $\dagger$ Theoretical Physics and Center for Biophysics, Saarland University, Saarbrücken, \\ Germany \\ $\ddagger$ Department of Physics, Faculty of Science, University of Helsinki, P.O. Box 64, FI-00014 \\ Helsinki, Finland \\ T These authors contributed equally to this work \\ E-mail: chetan.poojari@uni-saarland.de; jochen.hub@uni-saarland.de \\ Phone: +49 (0)681 302-2740. Fax: +49 (0)681 302-2748
}

\begin{abstract}
Itraconazole is a triazole drug widely used in the treatment of fungal infections, and it is in clinical trials for treatment of several cancers. However, the drug suffers from poor solubility, while experiments have shown that itraconazole delivery in liposome nanocarriers improves both circulation half-life and tissue distribution. The drug release mechanism from the nanocarrier is still unknown, and it depends on several factors including membrane stability against defect formation. In this work, we used molecular dynamics simulations and potential of mean force (PMF) calculations to quantify the influence of itraconazole on pore formation over lipid membranes, and we compared
\end{abstract}


the effect by itraconazole with a pore-stabilizing effect by the organic solvent dimethyl sulfoxide (DMSO). According to the PMFs, both itraconazole and DMSO greatly reduce the free energy of pore formation, by up to $\sim 20 \mathrm{~kJ} \mathrm{~mol}^{-1}$. However, whereas large concentrations of itraconazole of $8 \mathrm{~mol} \%$ (relative to lipid) were required, only small concentrations of few mol\% DMSO (relative to water) were sufficient to stabilize pores. In addition, itraconazole and DMSO facilitate pore formation by different mechanisms. Whereas itraconazole predominantly aids the formation of a partial defect with a locally thinned membrane, DMSO mainly stabilizes a transmembrane water needle by shielding it from the hydrophobic core. Notably, the two distinct mechanisms act cooperatively upon adding both itraconazole and DMSO to the membrane, as revealed by an additional reduction of the pore free energy. Overall, our simulations reveal molecular mechanisms and free energies of membrane pore formation by small molecules. We suggest that the stabilization of a locally thinned membrane as well as the shielding of a transmembrane water needle from the hydrophobic membrane core may be general mechanism by which amphiphilic molecules facilitate pore formation over lipid membranes at sufficient concentration.

\section{Introduction}

Itraconazole (ITZ, Fig 1A) is a first-generation lipophilic, triazole antifungal drug widely used to treat dermatologic diseases, aspergillosis, blastomycosis, coccidioidomycosis, histo-

plasmosis, penicilliosis and coccidioidomycosis. ${ }^{1-16}$ Apart from their antifungal activity, ITZ is currently in clinical trials for treatment of cancers such as esophageal cancer (Phase 2, ClinicalTrials.gov Identifier: NCT04018872), basocellular carcinoma of the skin (ClinicalTrials.gov Identifier: NCT03972748) and non-small cell lung cancer (Early Phase 1 completed, ClinicalTrials.gov Identifier: NCT02357836). Additionally, in vitro and in vivo studies have shown ITZ to inhibit influenza A virus by altering the cellular cholesterol concentration. ${ }^{17}$

ITZ belongs to azole class of antifungals and their broad application makes it a good start- 


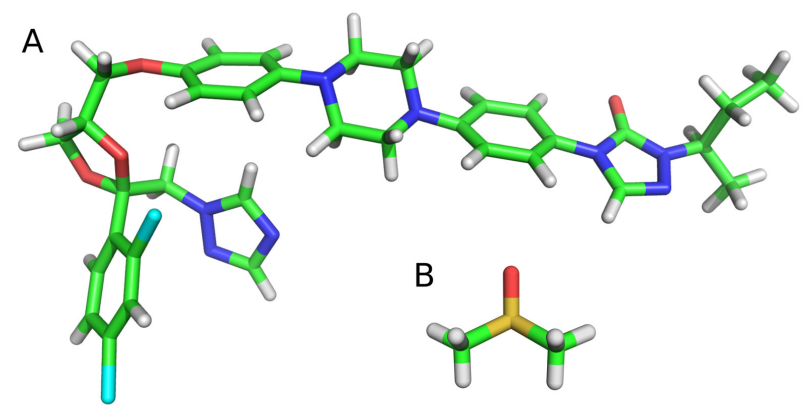

Figure 1: Stick representation of (A) itraconazole and (B) DMSO colored by element: carbon (green), oxygen (red), nitrogen (blue), chlorine (cyan), hydrogen (white), and sulfur (yellow).

ing structure for the design of new formulations to treat various diseases. The general mechanism of ITZ and triazoles is to inhibit the activity of cytochrome P450 51 (CYP 51), also known as lanosterol $14 \alpha$-demethylase. ${ }^{18}$ The enzyme catalyzes the removal of methyl group from lanosterol to produce ergosterol, a key component in the fungal cell membrane. ${ }^{19}$ Treatment with ITZ disrupts the synthesis of ergosterol and produces methylated sterols such as 14- $\alpha$-methylated lanosterol, 4,14-dimethylzymosterol, and 24-methylenedihydrolanosterol. ${ }^{20}$ The methyl sterols alter the fungal cell membrane properties, eventually leading to fungal cell death. ${ }^{1}$ In addition, CYP 51 is required for the synthesis of cholesterol in humans and other sterols in plants. ${ }^{21}$ Thus, CYP 51 can also be targeted in humans to decrease the cholesterol level.

Moreover, the metabolite of ITZ, hydroxy-itraconazole is also effective against fungal infections, thus, further increasing the potency of ITZ treatment. ${ }^{22}$ Being lipophilic, ITZ is distributed to a larger extent in the tissues of skin, nails, lungs, muscles, bones, kidney, and female genital tract than in plasma. ${ }^{22}$ The drug is administered in the form of capsules, as oral formulations, and intravenously. However, the therapeutic benefits of the drug are reduced due to its low solubility in water. ${ }^{23}$ To overcome the poor solubility and to increase the bioavailability of the drug, the oral and intravenous formulations are prepared in combination with hydroxypropyl- $\beta$-cyclodextrin (HP- $\beta$-CD) which is marketed as Sporanox. ${ }^{24}$ Unfortunately, the elimination of HP- $\beta$-CD is reduced by 6 -fold in patients with severe renal 
impairment. ${ }^{25}$ Thus, the need for oral and intravenous administration without the HP- $\beta$-CD formulation is important.

As an alternative, lipid-based drug delivery systems such as liposomes (spherical vesicles) are commonly used as drug carriers. The liposomes are non-toxic and can trap lipophilic drugs within the lipid bilayer and hydrophilic drugs in the central aqueous space. ${ }^{26}$ But these conventional liposomes are rapidly eliminated $(\sim 1 \mathrm{hr})$ from the bloodstream by the macrophages in liver and spleen, thus limiting its bioavailability. ${ }^{27,28}$ To improve the circulation half-life and stability, lipids in the liposomes are covalently linked to polyethylene glycol (PEG) chains which prevents rapid clearance from the blood stream and increases the circulation half-life to $1-2$ days. $^{27}$

Considering the benefits of using liposomes as drug carriers, several experiments have been designed to encapsulate ITZ in liposomes. Studies with PEGylated liposomes showed ITZ encapsulation efficiency to increase with low concentration, where $90 \%$ efficiency was reached with lipid concentration of $25 \mathrm{mg} / \mathrm{ml}$ and drug concentration at $0.3 \%(\mathrm{w} / \mathrm{w}) .{ }^{29} \mathrm{In}$ another study, tissue distribution was observed after loading ITZ into conventional uncoated liposomes composed of dipalmitoylphosphatidylcholine (DPPC) lipids. ${ }^{30}$ Intravenous administration of DPPC liposomes-ITZ showed high concentration of the drug in the infected tissues (lung, brain and liver) with higher in vivo efficacy when compared to formulation made of cyclodextrin. ${ }^{30}$ To further increase the circulation half-life and biodistribution of the drug, a study was performed with liposomes coated with carboxymethyl chitosan (CMCITZ-Lips). ${ }^{31}$ Intravenous administration of the formulation showed CMC-ITZ-Lips to have longer half-life when compared to ITZ in solution or when loaded in conventional uncoated liposomes. ${ }^{31}$

Despite in vivo experiments providing convincing evidence towards ITZ encapsulated in PEGylated liposome formulations to be effective and a gold-standard method in drug delivery, it remained unknown how ITZ partitions into conventional and PEGylated liposomes, and in liposomes containing cholesterol. Recently, using atomistic molecular dynamics (MD) 
simulations in combination with experimental techniques, structural and dynamic properties of the ITZ-liposomes system were studied. MD simulations were performed with ITZ payload in 1-palmitoyl-2-oleoyl-sn-glycero-3-phosphocholine (POPC) membranes varying in concentration from 3 mol\% to $16.6 \mathrm{~mol} \%{ }^{32}$ For comparison, membranes coated with 7 mol\% PEG chains were also simulated. In both non-pegylated and pegylated membranes, ITZ orients parallel to the membrane surface and occupies a position between the glycerols and the double-bonds of the POPC acyl chain. Nevertheless, ITZ had no or only little effects on the lipid structure. In addition, ITZ was also seen partitioning into PEG layer. Next, to evaluate the significance of cholesterol in liposomal-based drug delivery, simulations were performed with ITZ in POPC/cholesterol membranes. ${ }^{33}$ The simulations revealed ITZ and cholesterol to separate out in membranes, and fluorescence measurements showed ITZ to have reduced affinity for lipid membrane in presence of cholesterol. The findings suggest cholesterol not to be a suitable component of the formulation in liposome-based drug delivery. Further, MD simulations also revealed that when ITZ-membranes bilayers are loaded with 1,6-Diphenyl1,3,5-hexatriene (DPH) fluorescent probes, ITZ affects DPH orientation by pushing DPH to the membrane center. ${ }^{34}$ This further affects the fluorescence measurements of lipid order parameter and other dynamic parameters. Thus, MD simulations have become promising technique complementing experimental methods to gain structural insights into the dynamics of the molecules.

Although the advantages of using liposomal-based ITZ delivery are quite promising, very little is known about the ITZ release mechanism into the target tissues. Drug release pathways from nanocarriers can be grouped into the following four release mechanisms: ${ }^{35,36}$ a) diffusion, drug release due to the concentration gradient across the membrane, b) solvent influx, drug release driven by movement of solvent into the drug carrier, c) degradation, drug release by enzyme degradation of nanocarrier and d) stimuli, drug release due to electric field, sound, $\mathrm{pH}$, temperature, ionic strength etc. The release mechanisms depend on several factors, such as the properties of the drug molecule and the nanocarrier, the drug-lipid ra- 
tio, and the interactions between drug and carrier. The efficiency of different pathways are expected to be inversely correlated with membrane stability. Namely, increased propensity of the membrane for forming aqueous defects would render the membrane more sensitive to stimuli, and defects would increase solvent influx. In addition, membrane defects might render liposomes more fusogenic and ITZ more accessible to enzymes.

Therefore, we here used MD simulations and potential of mean force (PMF) calculations to quantify the effect of ITZ on the stability of a POPC model membrane. To this end, we computed the PMF of pore formation along a recently developed reaction coordinate (RC) for membranes with increasing ITZ content. ${ }^{37}$ The RC quantifies the connectivity of a polar defect over the membrane, hence referred to as 'chain coordinate' $\xi_{\mathrm{ch}}$, rendering the $\mathrm{RC}$ highly suitable to follow the nucleation of a pore. However, the $\mathrm{RC}$ is not designed to follow pore expansion (after pore nucleation) along increasing pore radii. We previously found that quantifying the connectivity is critical to avoid undesired hysteresis effects during PMF calculations of pore nucleation, as found for previously used RCs. ${ }^{38,39}$ Indeed, the chain coordinate resolved for the first time a nucleation barrier of pore formation over certain membranes, demonstrating that aqueous defects may be metastable (long-living). ${ }^{40}$ Such metastable pores have first been proposed based on memory effects observed during electroporation experiments in the 1970 s and 80 s. ${ }^{41,42}$ Henceforth, the RC was used to study pore formation by cationic polymers. ${ }^{43}$

To test whether the mechanism by which ITZ influences pore formation is more general among membrane-interacting molecules, we further studied the effect of dimethyl sulfoxide (DMSO, Fig 1B) on the free energies of pore formation at varying concentrations. DMSO is a commonly used organic solvent, capable of dissolving polar and apolar solutes. The effects of DMSO on lipid membranes have been studied in great detail by experimental and molecular dynamics studies. DMSO affects the structure, hydration properties, and phase behavior of lipid membranes. ${ }^{44-47}$ In addition, it is well established that DMSO increases the permeability of membranes for both non-electrolytes ${ }^{48}$ and ions. ${ }^{49,50}$ MD simulations 
revealed the molecular mechanisms underlying the DMSO effects on membrane structure, hydration, and permeability. ${ }^{51-54}$ It was found that DMSO molecules preferentially bind at the interface between the polar head groups and the apolar membrane core, thereby acting as a spacer between lipids and rendering membranes thinner and floppier. ${ }^{55}$ At higher DMSO concentrations, spontaneous formation of water defects has been observed in several simulation studies. ${ }^{50,55-57}$ These studies did not reveal the energetics of pore formation as studied here, but instead focused on the spontaneous formation of a pore by means of a large perturbation imposed by high DMSO content. Complementary, Lin et al. computed the PMF for the permeation of single water molecules in presence of low DMSO concentration, but this study did not consider continuous, membrane-spanning water defects. ${ }^{58}$

Interestingly, an increased permeation of ions over cellular membranes was found already at low DMSO concentrations of only few mol\% and, at reduced effects, even below 1 mol\%. ${ }^{49}$ Since ion permeation over membranes involves the formation of a water pore, these findings were interpreted as an increased propensity of the cellular membrane for the formation of pores in presence of DMSO. This suggests that DMSO may stabilize water defects over membranes at low concentration, at which only minor effects on the membrane structure were found. The mechanism by which DMSO stabilizes water defects at such low DMSO concentrations is not understood.

\section{Methods}

\section{Simulation setup and parameters}

The POPC, ITZ, and DMSO were described using the OPLS all-atom (OPLS-AA) force field. ${ }^{59}$ The POPC and ITZ force field parameters were taken from previous studies. ${ }^{32,60-62}$ Details on the ITZ parametrization are described in Ref. 32. For DMSO, the default parameters provided in the OPLS-AA force field package were used. Water was described by the TIP3P model. ${ }^{63}$ The initial membranes of pure POPC or POPC loaded with ITZ were 
prepared with the MemGen ${ }^{64}$ server, which generates a symmetric lipid distribution. All systems contained a total of 162 molecules of POPC plus ITZ. Each system was solvated with 35 TIP3P water molecules per lipid. We studied four different systems: POPC only, as well as POPC loaded with 4, 6, or 12 ITZ molecules, corresponding to a molar fraction (relative to lipid) of approx. $2.5 \%, 3.8 \%$, and $8 \%$.

The solvated POPC and POPC/ITZ systems were first subjected to energy minimization with steepest-descent algorithm to remove atomic clashes. Next, the systems were equilibrated under NVT conditions for $1 \mathrm{~ns}$. Here, the temperature of $300 \mathrm{~K}$ was regulated by coupling water and POPC/ITZ separately to v-rescale thermostat with a time constant $\left(\tau_{t}\right)$ of 0.1 ps. ${ }^{65}$ Following NVT equilibration, the systems were equilibrated under NPT conditions for $10 \mathrm{~ns}$, here we switched to the Nose-Hoover thermostat $\left(\tau_{p}=0.5 \mathrm{ps}\right)^{66,67}$ to regulate the temperature. The pressure was kept at 1 bar by coupling to a semi-isotropic ParrinelloRahman barostat $(\tau=2 \mathrm{ps}){ }^{68}$ The geometry of water molecules was constrained with the SETTLE algorithm, ${ }^{69}$ and all other bonds were constrained with LINCS. ${ }^{70}$ The Coulombic interactions were computed using the particle-mesh Ewald method. ${ }^{71,72}$ During NVT and NPT equilibrations runs, the ITZ and P atoms of the lipids were position restrained with a force constant of $1000 \mathrm{~kJ} \mathrm{~mol}^{-1} \mathrm{~nm}^{-2}$ and $500 \mathrm{~kJ} \mathrm{~mol}^{-1} \mathrm{~nm}^{-2}$ respectively. Following equilibration, final production MD runs without any position restraints were run for a $250 \mathrm{~ns}$ with an integration time step of $2 \mathrm{fs}$.

Systems with DMSO were built from an equilibrated POPC system by inserting DMSO molecules at random positions using the GROMACS module gmx insert-molecules. Overlapping water molecules were removed. Systems with DMSO/water molar ratios of $2.5 \%$, $5 \%, 10 \%$, and $15 \%$ were constructed, composed of the following number of $\mathrm{DMSO} /$ water molecules, respectively: 132/5283, 248/4950, 450/4470,612/4085. These systems contained 162 POPC molecules. In addition, to study the combined effect of DMSO and ITZ, we inserted $5 \mathrm{~mol} \%$ DMSO (relative to water) into the equilibrated system with $8 \mathrm{~mol} \% \mathrm{ITZ}$ (relative to lipid), giving a system with the following number of DMSO/ITZ/water molecules: 
248/12/4958. After energy minimization with the steepest-descent algorithm, the systems were equilibrated without any position restraints for at least $150 \mathrm{~ns}$. We found that the individual DMSO molecules entered and left the membrane multiple times within this simulation time, suggesting that the DMSO distributions were fully equilibrated. All other parameters were identical to the POPC/ITZ simulations.

Simulations were performed on an in-house modified version of GROMACS 2018.3, ${ }^{73}$ which implements the $\xi_{\text {ch }}$ coordinate. ${ }^{37}$ The membrane thicknesses presented in (Fig. 5) were computed from the last $200 \mathrm{~ns}$ and last $100 \mathrm{~ns}$ of the ITZ- and DMSO-containing simulations, respectively. Here, the thickness was defined as the average distance between the phosphate atoms of the two membrane leaflets.

\section{PMF calculations}

PMFs of pore formation were computed along a recently developed reaction coordinate, referred to as 'chain coordinate' $\xi_{\mathrm{ch}}$, which probes the connectivity of polar atoms in the defect. ${ }^{37,39}$ The $\xi_{\text {ch }}$ coordinate is unitless and defined between 0 and unity, where a small value $(\approx 0.25)$ indicates the planar, unperturbed membrane, and a value close to unity indicates a continuous polar defect. In brief, the $\xi_{\text {ch }}$ is defined using a membrane-spanning cylinder that is decomposed into $N_{s}$ slices. Then, $\xi_{\text {ch }}$ is given by the fraction of slices that are occupied by polar atoms. The lateral position of the cylinder in the membrane plane is dynamically defined, allowing the cylinder to 'follow' the defect. This property is critical to avoid that the system moves along $\xi_{\mathrm{ch}}$ by shifting the defect laterally out of the cylinder. In this study, we used a cylinder with radius $R_{\text {cyl }}=1.2 \mathrm{~nm}$, composed of 28 slices with a thickness of $0.1 \mathrm{~nm}$ each. Critically, $R_{\text {cyl }}$ is not the radius of the polar defect; instead, $R_{\text {cyl }}$ defines the transmembrane volume considered to quantify the connectivity of the polar defect. Defining

the RC with such a cylinder excludes the misinterpretation of two laterally displaced partial defects connected with the upper and lower water reservoir, respectively, as a continuous transmembrane defect. The saturation of a slice upon the addition of the first polar atom 
was taken as $\zeta=0.75$. As polar atoms contributing to $\xi_{\mathrm{ch}}$, we used the oxygen atoms of water, oxygen atoms of lipid phosphate groups, as well as the carbonyl oxygen atoms of the ester groups.

The PMFs were computed using umbrella sampling. The initial frames were generated by gradually pulling the system along $\xi_{\text {ch }}$ within $100 \mathrm{~ns}$, allowing us to extract frames for umbrella windows. For systems with ITZ, 37 windows were used, centered at $\xi_{\text {ch }}=(0.065$ 0.1450 .2250 .3050 .3850 .4650 .5450 .6250 .640 .660 .680 .700 .720 .740 .760 .780 .800 .810 .82 $0.830 .840 .850 .860 .870 .880 .890 .900 .910 .920 .930 .940 .950 .960 .970 .980 .991 .0)$. For systems with DMSO, 27 windows were used, centered at $\xi_{\mathrm{ch}}=(0.0650 .1450 .2250 .3050 .385$ $\begin{array}{llllllllllllllllll}0.465 & 0.545 & 0.625 & 0.64 & 0.66 & 0.68 & 0.70 & 0.72 & 0.74 & 0.76 & 0.78 & 0.80 & 0.82 & 0.84 & 0.86 & 0.88 & 0.90 & 0.92\end{array}$ 0.940 .960 .981 .0 ). An umbrella force constant of $5000 \mathrm{~kJ} \mathrm{~mol}^{-1} \mathrm{~nm}^{-2}$ was used in windows at $\xi_{\mathrm{ch}}<0.7$ and $10000 \mathrm{~kJ} \mathrm{~mol}^{-1} \mathrm{~nm}^{-2}$ in all other windows. Windows were simulated for $300 \mathrm{~ns}$ and $100 \mathrm{~ns}$ for ITZ- and purely-DMSO-containing systems, respectively, where the first $50 \mathrm{~ns}$ and $20 \mathrm{~ns}$ were removed for equilibration. PMFs for pure POPC using the Berger or Slipids force field, use 37 windows, 100 ns of simulations per window, and removing the first $10 \mathrm{~ns}$ for equilibration. By computing PMFs from various time blocks, we found that the equilibration times were sufficient. Simulation parameters were identical to the unbiased simulations. The PMFs were derived with the weighted histogram analysis method (WHAM), as implemented in the gmx wham ${ }^{74}$ module. Statistical uncertainties were estimated using the Bayesian bootstrap of complete histograms.

\section{Results}

\section{Influence of the force field on the PMF of pore formation}

To build upon recent simulation studies on ITZ that used the OPLS-AA force field for ITZ/POPC system, ${ }^{32-34}$ we chose to apply the OPLS-AA force field for the present study as well. Hence, we first evaluated the role of the applied OPLS-AA force field on the free 


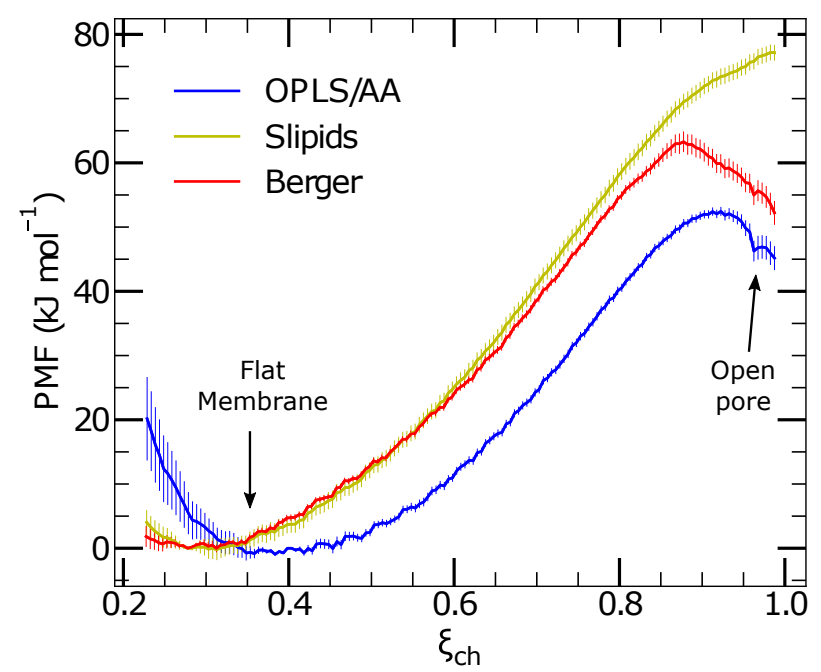

Figure 2: PMF of pore formation over a POPC membrane, computed with the Slipids, OPLS-AA, and Berger et al. force fields.

energies of pore formation over a pure POPC membrane. In our previous studies, ${ }^{37,40}$ we compared PMFs computed using Charmm36, ${ }^{75}$ Slipids ${ }^{76}$ and Berger force fields. ${ }^{77}$ We found good agreement between Charmm36 and Slipids for various phosphatidylcholine (PC) lipids as PMFs differed by only $\sim 5 \mathrm{~kJ} \mathrm{~mol}^{-1} .{ }^{40}$ For dimyristoylphosphatidylcholine (DMPC), we found in addition good agreement with the Berger force field, although the Berger force field is slightly more prone to form metastable (long-living) open pores as compared to Charmm36 and Slipids. ${ }^{37}$

Fig. 2 compares the PMF of pore formation for a patch of 162 POPC lipids, computed with the Slipids, OPLS-AA, and Berger force fields. Here, the minimum at $\xi_{\mathrm{ch}} \approx 0.4$ for OPLS-AA and at $\xi_{\mathrm{ch}} \approx 0.3$ for Slipids or Berger corresponds to the flat, unperturbed membrane, while $\xi_{\mathrm{ch}} \approx 1$ corresponds to the open pore. The maximum at $\xi_{\mathrm{ch}} \approx 0.9$ found in the PMFs computed with Berger or OPLS-AA indicates the transition state of pore formation, characterized by the presence of a membrane-spanning thin water needle (Fig. 2, red and green; see also Ref. 40). Evidently, the force field has a larger effect on the PMFs for POPC membranes as compared to the previously studied DMPC membranes. ${ }^{37}$ First, both the Berger and OPLS-AA force fields yield a free energy barrier for pore formation, implying that the open pore represents a long-living, metastable state. In contrast, the Slipids force 
field yields no barrier, implying that the open pore is an unstable state, which would rapidly close within a few tens of nanoseconds in a free simulations. In addition, the PMFs suggest varying free energy differences $\Delta G_{\text {pore }}$ between the open pore $\left(\xi_{\text {ch }}=0.95\right)$ and the flat membrane of approx. 75,55 , and $47 \mathrm{~kJ} \mathrm{~mol}^{-1}$ for Slipids, Berger, and OPLS-AA. respectively. In addition, the position of the minimum of the PMF corresponding to the flat, unperturbed membrane differs among the three force fields. Because $\xi_{\text {ch }}$ is defied by the fraction of cylinder slices that are occupied by polar atoms, this difference merely reflects that the POPC membrane modeled with OPLA/AA is slightly thinner as compared to Slipids and Berger et $a l$.

Taken together, both the shape of the PMF as well as the free energy cost $\Delta G_{\text {pore }}$ of pore formation are quite force field-dependent for POPC membranes. Because experimental data on $\Delta G_{\text {pore }}$ are to our knowledge not available, we cannot resolve at present which force field is correct and which is wrong. The PMFs reported in Figs. 3 and $7 \mathrm{~A}$ are computed with the OPLS-AA force field, which yields the lowest free energy of pore formation for pure POPC. Hence, we note that the $\Delta G_{\text {pore }}$ values reported below for membranes containing ITZ or DMSO are likewise influenced by the force field.

\section{High concentration of ITZ greatly facilitates pore formation}

To quantify the effect of ITZ on pore formation, we computed the PMF of pore formation over a POPC membrane containing 0, 2, 4, or 8 mol\% ITZ (Fig. 3). Figure 4A-F presents typical simulation snapshots during the formation of the pore, taken from the final snapshots of the umbrella sampling simulations with $8 \mathrm{~mol} \%$ ITZ. The PMFs reveal that ITZ reduces the height of the free energy barrier $\Delta G_{\mathrm{bar}}$ as well as the free energy of the open pore $\Delta G_{\text {pore }}$ in a concentration-dependent manner (Fig. 3). Namely, the addition of 2 or 4 mol\% ITZ leads to a moderate reduction of $\Delta G_{\mathrm{bar}}$ by $\sim 6 \mathrm{~kJ} \mathrm{~mol}^{-1}$, while $8 \mathrm{~mol} \%$ ITZ leads to a large reduction of $\Delta G_{\mathrm{bar}}$ by $\sim 22 \mathrm{~kJ} \mathrm{~mol}^{-1}$. Critically, and in contrast to the effect of DMSO discussed below, ITZ facilitates the formation of a partial defect with a locally thinned membrane, as evident 


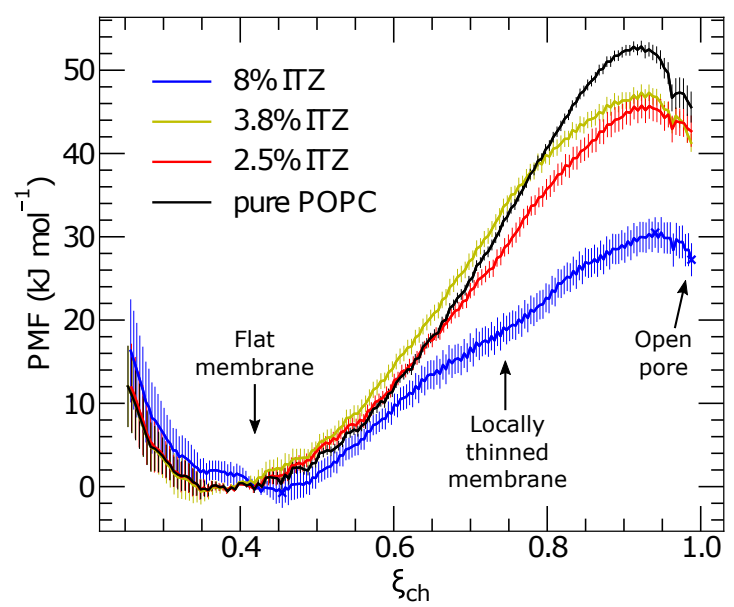

Figure 3: PMF of pore formation over a POPC membrane, containing $0,2.5,3.8$, or 8 mol\% ITZ (for color code, see legend).

from a reduced slope of the PMF at $\xi_{\text {ch }}<0.75$ (Fig. 3, blue curve, and Fig. 3B).

Two mechanisms for the ITZ effect on the free energies are conceivable; first, an indirect effect on pore formation by modifying the overall membrane properties, and second, by direct interactions of ITZ with the aqueous defect. According to the first mechanism, insertion of the drug might render the membrane thinner, thereby facilitating the formation of pores. It was previously found that the free energy of pore formation strongly correlates with membrane thickness, as shown by comparing membranes of PC lipids with increasing tail lengths. ${ }^{37,40,79,80}$ However, we found that the addition of ITZ does not lead to thinning of the membrane (Fig. 5A), but instead even leads to slightly thicker membranes at 3.8 and $8 \mathrm{~mol} \% \mathrm{ITZ}$. Hence, it is unlikely that ITZ facilitates pore formation via such an indirect effect.

Instead, visual inspection of the simulations (Fig. 4), as well as the analysis of the densities of head group, water, tails, and ITZ (Fig. 6), revealed binding of ITZ to thinned membrane and to the water defect, strongly suggesting that ITZ stabilizes the pore by direct interactions. Here, the densities were computed as the average during four representative umbrella sampling windows corresponding to a flat membrane, a thinned membrane, the state with a thin water needle, and the open pore. In the planar, unperturbed membrane, in 

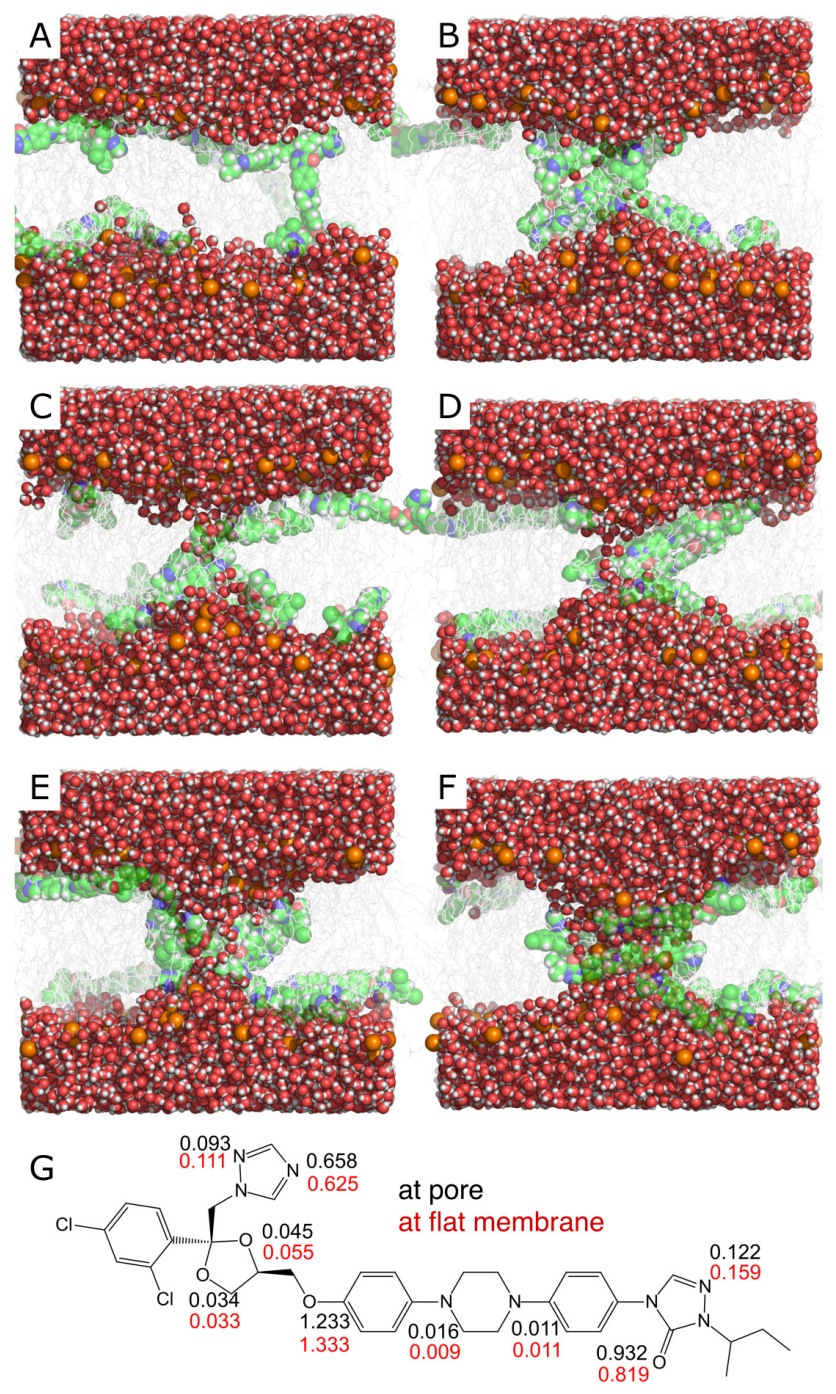

Figure 4: (A-F) Typical simulations snapshots of the emerging pore, taken from the final snapshots of umbrella sampling windows with $8 \mathrm{~mol} \%$ ITZ. Panels A-F correspond to windows restrained at $\xi_{\mathrm{ch}}=0.385,0.74,0.82,0.84,0.92$, or 1.0 , respectively. Water is shown as red/white spheres, ITZ as colored transparent spheres, lipid phosphate atoms as orange spheres, and other lipid atoms as transparent light grey sticks. (G) Average number of hydrogen bonds formed by polar nitrogen and oxygen atoms of ITZ bound to the pore (black) or bound to the flat membrane (red).

line with previous simulations, ${ }^{32-34}$ ITZ predominantly takes a horizontal conformation (parallel to the membrane plane, Figs. $4 \mathrm{~A}$ and $6 \mathrm{~A}$ ) where it can form polar interactions with the lipid head group region and hydrophobic contacts with the tail region. As the membrane is thinning, ITZ is greatly enriched at the emerging defect (Figs. 4B and 6B). This enrichment of ITZ facilitates membrane thinning, as evident from the reduced slope of the PMF in this 

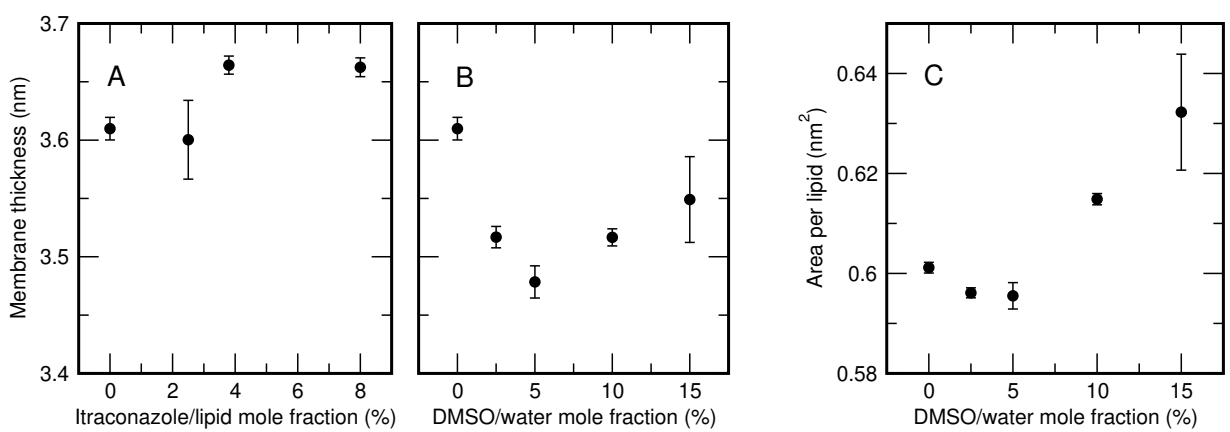

Figure 5: Thickness of POPC membrane quantified as the average distance of phosphate atoms from opposite leaflets, containing (A) ITZ or (B) DMSO. ITZ and DMSO concentrations are given as mole fraction relative to the lipid and to the water, respectively, as indicated by the axis labels. (C) Area per lipid in simulations containing DMSO. Statistical errors were computed by binning analysis. ${ }^{78}$

region (Fig. 3, $\xi_{\text {ch }}<0.75$ ). As a water needle and, subsequently, the open pore form, ITZ remains strongly enriched at the defect, albeit to slightly reduced magnitude as compared to the state with a thinned membrane (Fig. 4C-F and Fig. 6C/D). Together, this suggests that ITZ facilitates pore formation predominantly by stabilizing a state with a thinned membrane, and to a lower degree by shielding the water needle from the hydrophobic core of the membrane. Interestingly, these trends observed for ITZ seem inverted as compared to the effects for DMSO discussed below.

What drives ITZ to the defect? To characterize the interactions of ITZ with the defect in atomic detail, we analyzed the average number of hydrogen bonds formed by polar nitrogen and oxygen atoms of ITZ, as summarized in Fig. 4G. Here, the number of hydrogen bonds were taken from an umbrella window with an open pore, and the numbers were obtained for ITZ in contact with the pore (Fig. 4G, black numbers) and for ITZ in contact with a patch of flat membrane (Fig. 4G, red numbers). Evidently, the hydrogen bond numbers differ only marginally, demonstrating that the ITZ enrichment at the pore cannot be rationalized by a direct polar ITZ-pore interactions. In such cases, it is difficult to pin down the driving force as it may be complex combination of competing interaction as well as entropic effects. However, we speculate that an indirect effect by the lipid packing may play a role; in the flat membrane ITZ may perturb the lipid packing, and this perturbation may be partly relieved 


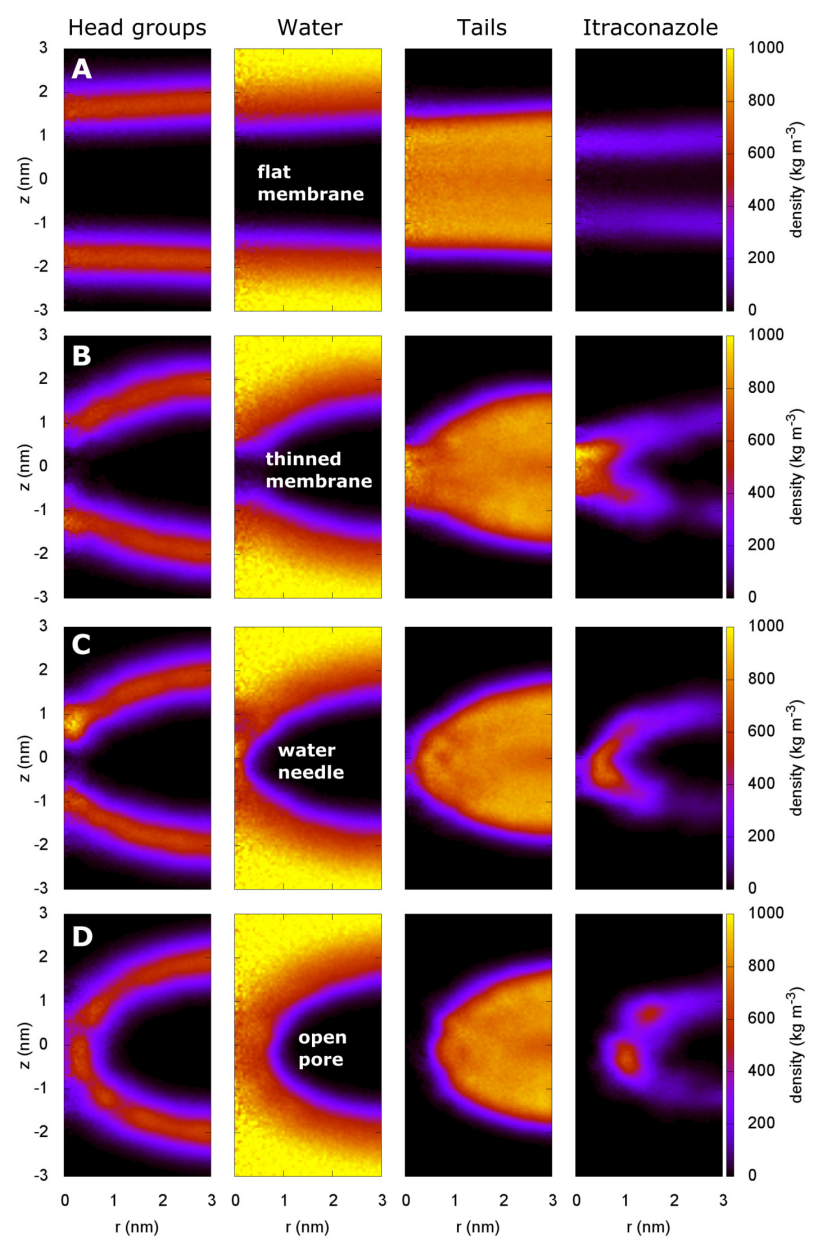

Figure 6: Mass densities during pore formation, plotted as the radial distance $r$ and vertical distance $z$ from the pore center. From left to right column: lipid head groups, water, lipid tails, and ITZ (see labels on top the columns). (A) Planar, unperturbed membrane $(\xi=0.305)$, (B) thinned membrane $(\xi=0.76)$, (C) water needle $(\xi=0.89)$ (D) fully formed pore $(\xi=1.0)$. Evidently, ITZ binds to emerging or fully formed pores.

upon ITZ enrichment at the defect. Conversely, the entropic cost of demixing ITZ and lipid disfavors ITZ enrichment at the pore, rationalizing why high ITZ concentrations are required to ITZ enrichment and pore formation (cf. Fig. 3).

\section{Effect of DMSO on free energies of pore formation}

To rationalize the DMSO effect on membrane pores in energetic terms, we computed the PMFs of pore formation over a POPC membrane in presence of DMSO concentrations between 0 and $15 \mathrm{~mol} \%$ (relative to water). Further, we aimed to clarify whether the stabi- 

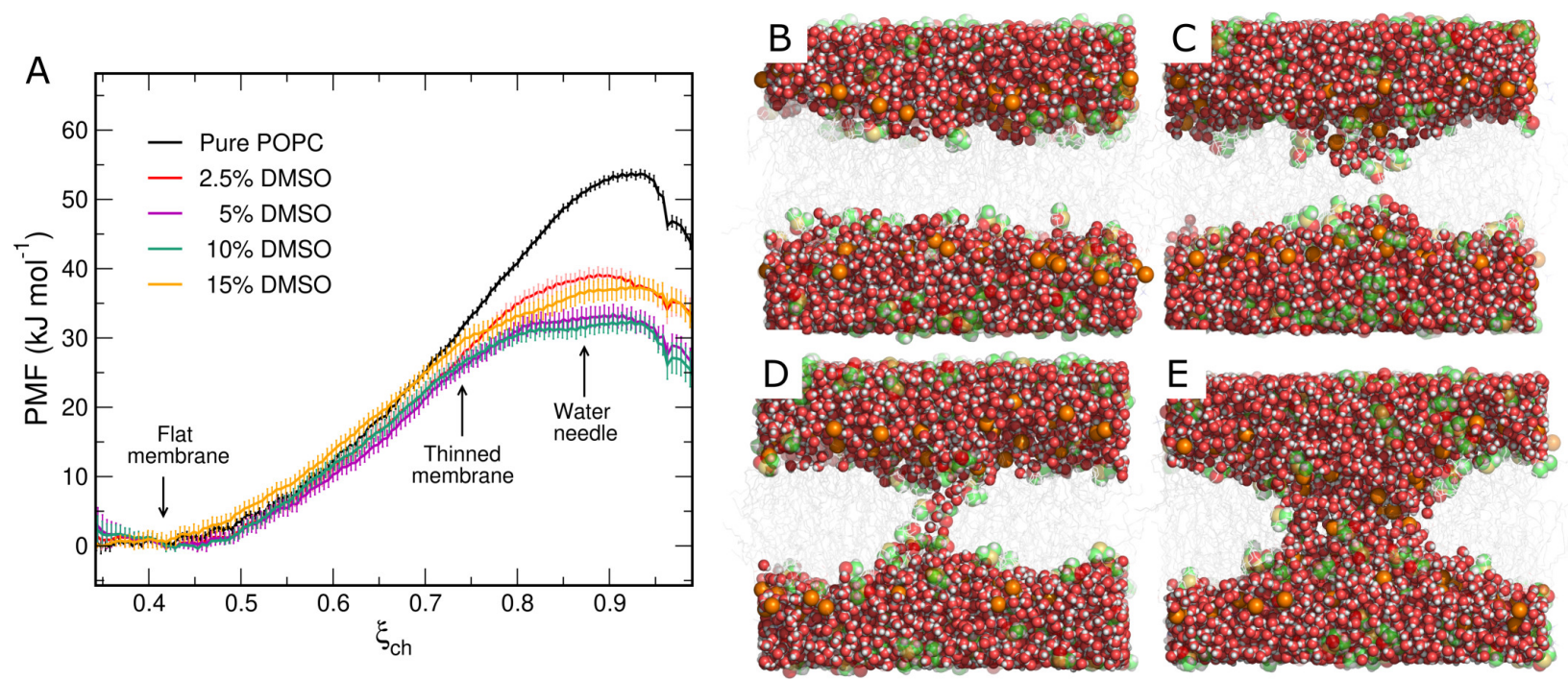

Figure 7: (A) PMFs for pore formation over a POPC membrane at DMSO mole fractions of $0,2.5,5,10$, and $15 \mathrm{~mol} \%$ relative to the water concentration (see legend). (B-E) Typical simulations snapshots of the emerging pore, taken from the final snapshots of umbrella sampling windows with 5 mol\% DMSO. (B) Flat membrane, (C) thinned membrane, (D) water needle, (E) open pore. Panels B-E correspond to windows restrained at $\xi_{\text {ch }}=0.385,0.74$, $0.82,0.98$, respectively. Water is shown as red/white spheres, DMSO as colored transparent spheres, lipid phosphate atoms as orange spheres, and other lipid atoms as transparent light grey sticks.

lization of the thinned membrane and of the aqueous defect by direct interactions with a small solute, as found for ITZ above, could be a common mechanism for other membraneactive compounds such as DMSO. Fig. 7A presents the PMFs for pore formation for POPC membranes with 2.5, 5, 10, and 15 mol\% DMSO, compared with the PMF for pure POPC. Evidently, DMSO greatly reduces the free energy barrier $\Delta G_{\text {bar }}$ for pore formation from $54 \mathrm{~kJ} \mathrm{~mol}^{-1}$ to values between 39 and $32 \mathrm{~kJ} \mathrm{~mol}^{-1}$. With transition state theory, these changes of $\Delta G_{\text {bar }}$ correspond to an increased rate of pore formation by factors between 400 and 6700 . Surprisingly, the stabilization of the open pore did not correlate with increased DMSO concentration on the range of 2.5 to $15 \mathrm{~mol} \%$. Instead, the PMFs for 2.5, 5, 10, and $15 \mathrm{~mol} \%$ DMSO are rather similar, with slightly more pronounced effects at 5 and $10 \mathrm{~mol} \%$, and slightly reduced effects at 2.5 and $15 \mathrm{~mol} \%$. Overall, these findings demonstrate that only 2.5 to $5 \mathrm{~mol} \%$ DMSO were sufficient for a major effect on pore formation.

In line with the analysis for ITZ presented above, we investigated whether DMSO stabi- 
lizes pores by modulating the membrane structure or by direct interactions with the pore. In agreement with previous studies, we found that the addition of large amounts of DMSO leads to an increased area per lipid $\left(A_{\mathrm{L}}\right)$; however only at a concentration of $\geq 10 \mathrm{~mol} \%$ (Fig. 5C), whereas at 2.5 or $5 \mathrm{~mol} \%$ DMSO $A_{\mathrm{L}}$ was even slightly decreased. This suggests that $A_{\mathrm{L}}$ does not correlate with free energy of pore formation, further implying that the $A_{\mathrm{L}}$ does not explain modulations of $\Delta G_{\mathrm{bar}}$.

In contrast, modulations of the membrane thickness clearly correlate with $\Delta G_{\text {bar }}$, as evident from decreased thicknesses upon addition of DMSO (Fig. 5B). For instance, in our simulations, $5 \mathrm{~mol} \%$ DMSO reduced the thickness of the membrane by $\sim 1.3 \AA$. Interestingly, the reduced thickness at $5 \mathrm{~mol} \%$ DMSO (Fig. 5B) did not impose an increased area per lipid (Fig. 5C), implying a reduced effective volume per lipid, possibly by decreasing the degree of hydration of the head groups. Previous studies with lipids of different tail length revealed a strong effect of membrane thickness on free energies of pore formation; ${ }^{37,40,79,80}$ however, in the light of these studies, from a reduced membrane thickness by $\sim 1.3 \AA$, as here found in simulations at $5 \mathrm{~mol} \% \mathrm{DMSO}$, we would expect a reduction of $\Delta G_{\text {bar }}$ by at most $10 \mathrm{~kJ} \mathrm{~mol}^{-1}$. Hence, reducing the thickness of the flat membrane does not fully explain the observed reduction of $\Delta G_{\text {bar }}$ by $26 \mathrm{~kJ} \mathrm{~mol}^{-1}$, suggesting that direct interactions of DMSO with the defect play in important role as well.

Indeed, an effect by DMSO via direct interactions with the defect is compatible with the representative snapshots of the simulation system with 5 mol\% DMSO shown in Fig. 7B-E, taken from the final simulation time of four umbrella sampling windows. Evidently, as the aqueous defect forms from a partial defect with a thinned membrane (Fig. 7C), over a transmembrane water needle (Fig. 7D) to a fully established pore (Fig. 7E), DMSO frequently forms contact with the defect. Interestingly, the effect of DMSO on the shape of the PMF differs from the effect of ITZ, pointing towards a different pore-stabilizing mechanism. Whereas ITZ at high concentration flattened the PMF at $\xi_{\text {ch }}<0.75$ as a consequence of binding to the thinned membrane, the PMFs are hardly altered by DMSO in this region. 


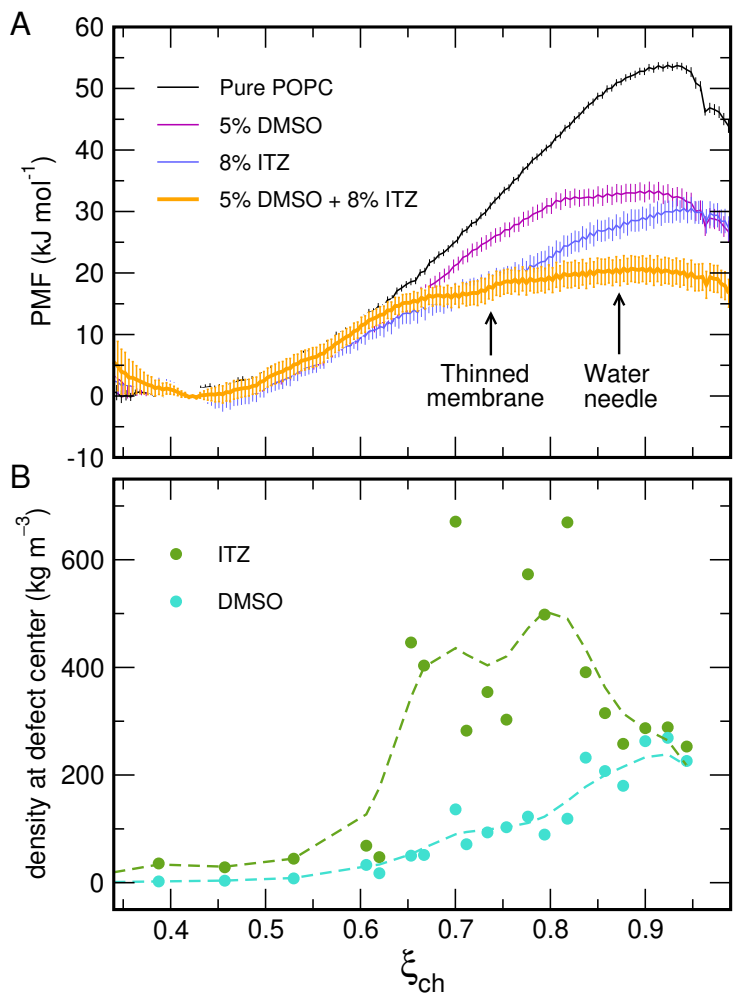

Figure 8: (A) PMFs for pore formation with 8 mol\% ITZ plus 5 mol\% DMSO (orange curve). For reference, PMFs are shown for pure POPC (black), 5 mol\% DMSO (magenta), and 8 mol\% ITZ (blue). (B) Density of ITZ (dark green) and DMSO (dark cyan) at the defect center during pore formation with $8 \mathrm{~mol} \%$ ITZ plus $5 \mathrm{~mol} \%$ DMSO, taken from the umbrella sampling windows used to compute the PMF in panel A. Here, the center of the defect was taken as a cylinder with hight $0.5 \mathrm{~nm}$ and radius $1 \mathrm{~nm}$ around the defect center. To guide the eye, dashed lines show the densities smoothed along $\xi_{\text {ch }}$ with a Gaussian filter $(\sigma=0.03)$. The respective two-dimensional densities are shown in Movie S1.

Instead, DMSO reduces the PMF mostly in the region where the water needle penetrates the hydrophobic membrane core at $0.8 \lesssim \xi_{\text {ch }} \lesssim 0.9$ (see Fig. 7A, arrows). This suggests that DMSO aids pore formation predominantly by shielding the water needle from the hydrophobic core, and only to a lower degree by stabilizing a thinned membrane, in contrast to the effect of ITZ.

\section{Cooperative effects of ITZ and DMSO on pore formation}

Because ITZ supports pore formation predominantly by stabilizing the thinned membrane, whereas DMSO by stabilizing the water needle, we asked whether these distinct effects can 
be combined. To this end, we computed the PMF over a POPC membrane in the presence of $8 \mathrm{~mol} \%$ ITZ (relative to lipid) plus $5 \mathrm{~mol} \%$ DMSO (relative to the water), presented in Fig. 8A (orange curve). Indeed, combining ITZ and DMSO stabilizes the open pore by another $10 \mathrm{~kJ} / \mathrm{mol}$ relative to the effect of each of the molecules alone. Interestingly, the shape of the PMF for the combined ITZ/DMSO reveals the pore-stabilizing effects specific to ITZ or DMSO alone: the PMF for ITZ/DMSO exhibits a reduced slope during membrane thinning, in line with the PMF for ITZ (Fig. 8, compare orange with blue, $\xi_{\text {ch }} \lesssim 0.75$ ). In addition, the PMF for ITZ/DMSO is nearly flat at the region of water needle formation, in line with the PMF for DMSO (Fig. 8, compare orange with magenta, $0.8 \lesssim \xi_{\text {ch }} \lesssim 9$ ). These findings demonstrate that the pore-stabilizing effects specific to DMSO and ITZ are indeed cooperative.

Additional evidence for the distinct effects of ITZ and DMSO is given by the densities of ITZ and DMSO at the pore, visualized in Movie S1. Each frame of the movie presents the average density of one umbrella window, decomposed into contributions from head groups, water, tails, ITZ, and DMSO. For reference, the average $\xi_{\mathrm{ch}}$ of the respective umbrella window is shown in the top left corner of the movie frames. Further, the average density of ITZ and DMSO at the pore center are shown in Figure 8B. The densities confirm that ITZ is mostly enriched at the thinned membrane, and only to a lower degree at the water needle. This trend is inverted for DMSO, which is mainly enriched at the water needle, and only to a lower degree at the thinned membrane.

\section{Discussion}

We used PMF calculations along a recently developed reaction coordinate ${ }^{37,39}$ to quantify the effect of two highly different molecules on pore formation over a POPC membrane. We found that both molecules, the antifungal ITZ and the organic solvent DMSO, facilitate pore formation, as evident from strongly reduced free energies of the open pore and strongly 
reduced free energy barriers for pore formation. However, the simulations suggest that these two molecules act predominantly by distinct mechanisms. ITZ mainly facilitates the formation of a partial defect with a locally thinned membrane, characterized by a reduced distance between the opposite head groups, but where water does not yet penetrate the hydrophobic core. In contrast, DMSO mainly facilitates the formation of a membranespanning water needle, presumably by partly shielding the water needle from the hydrophobic membrane core. ${ }^{50}$ The fact that ITZ and DMSO act by distinct mechanisms rationalizes that these mechanics act cooperatively upon adding both ITZ and DMSO to the membrane. However, we stress that the mechanisms of ITZ and DMSO are not exclusive; the PMFs show that ITZ also stabilizes a water needle and DMSO slightly facilitates membrane thinning, albeit to a lower degree (Fig. 6 and Movie S1). In addition to such effects by direct interaction with the defect, DMSO, but not ITZ, facilitates pore formation by reducing the thickness of the flat, unperturbed membrane; for the concentrations studied here, however, reducing the membrane thickness seems to play only a minor role as compared to the direct interactions with the defect.

Clearly, an experimental validation of the free energies and the cooperative effects found here would be highly desirable. Because it is difficult to measure the free energies of pores directly, assays that probe the leakage of membranes with respect to ions or polar molecules might be useful to validate our simulations.

How ITZ is released from liposome carriers is unknown. For several putative release pathways, the formation of membrane defects may represent a critical step, such as pathways involving stimuli (electric fields, sound etc.) or membrane fusion. Our results suggest that such pathways may be more accessible owing to the pore-stabilizing properties of ITZ, given that ITZ is present at sufficient concentrations. The cooperative effects of ITZ and DMSO suggest that such pathways might be further stimulated by the application of additional membrane-active small molecules similar to DMSO to the membrane.

We found that DMSO strongly facilitates pore formation at relatively low concentrations. 
Because the permeation of ions requires the formation of an aqueous defect, these findings imply that DMSO increase membrane permeability for ions at low DMSO concentrations. These results are in line with $\mathrm{He}$ et al. who reported increased $\mathrm{Ti}^{+}$and $\mathrm{Ca}^{2+}$ influx into CHO-K1 cells across unspecific pores upon application of DMSO. ${ }^{49}$ However, our results seem to contrast findings by de Ménorval et al. who found increased $\mathrm{Ca}^{2+}$ influx into cells only at high DMSO concentrations of 15 to $20 \mathrm{~mol} \% .{ }^{50}$ Hence, additional experimental data, possibly based on well-controlled model membranes, will be needed to validate the effects of DMSO on pores at low DMSO concentrations.

Many previous MD studies used large perturbations to induce pores, for instance by applying larger electric fields or large concentrations of DMSO. ${ }^{55,56,81-83}$ In addition, for thin dilauroylglycerophosphocholine (DLPC) membranes, also spontaneous pore formation over unperturbed bilayers has been reported. ${ }^{79}$ These studies showed that pores form by first penetrating the membrane with a thin water needle, subsequently followed by lipid head groups entering the membrane core to form a toroidal pore. PMF calculations confirmed this sequence of events during pore formation. ${ }^{37,40}$ However, the insights into free energies revealed that a membrane-spanning water needle does not represent a metastable intermediate state, as occasionally proposed, but that the water needle represents the transitions state (a free energy saddle point) in membranes that form metastable pores. Only as the pore grows to a diameter spanned by a few water molecules, the pore may be metastable. ${ }^{37,40}$ Here, we found that small molecules do not change this overall mechanism; namely, also in presence of ITZ and DMSO, a water needle forms, corresponding to the free energy barrier in the PMFs (Figs. 4D and 7C), before a wider defect is established (Figs. 4E/F and 7D). However, small molecules may drastically change the free energy landscape of pore formation, predominantly by aiding local membrane thinning and by shielding the defect from the hydrophobic membrane core. 


\section{Acknowledgement}

G.K. and C.P. were supported by the Deutsche Forschungsgemeinschaft via grants SFB 803/A12 and SFB 1027/B7, respectively.

\section{References}

(1) Lass-Flörl, C. Triazole antifungal agents in invasive fungal infections: a comparative review. Drugs 2011, 71, 2405-2419.

(2) Lestner, J.; Hope, W. W. Itraconazole: an update on pharmacology and clinical use for treatment of invasive and allergic fungal infections. Expert Opin. Drug Metab. Toxicol. 2013, 9, 911-926.

(3) Van Cutsem, J.; Van Gerven, F.; Van de Ven, M. A.; Borgers, M.; Janssen, P. A. Itraconazole, a new triazole that is orally active in aspergillosis. Antimicrob. Agents Chemother. 1984, 26, 527-534.

(4) Denning, D. W. Therapeutic Outcome in Invasive Aspergillosis. Clin. Infect. Dis. 1996, 23, 608-615.

(5) Amato, V. S.; Padilha, A. R.; Nicodemo, A. C.; Duarte, M. I.; Valentini, M.; Uip, D. E.; Boulos, M.; Neto, V. A. Use of itraconazole in the treatment of mucocutaneous leishmaniasis: A pilot study. Int. J. Infect. Dis. 2000, 4, 153-157.

(6) Sharkey-Mathis, P. K.; Velez, J.; Fetchick, R.; Graybill, J. R. Histoplasmosis in the acquired immunodeficiency syndrome (AIDS): treatment with itraconazole and fluconazole. J. Acquir. Immune Defic. Syndr. 1993, 6, 809-819.

(7) Kreisel, W.; Kochling, G.; Schilling, C. v.; Azemar, M.; Kurzweil, B.; Dölken, G.; Lindemann, A.; Blum, U.; Windfuhr, M.; Muller, J. Therapy of invasive aspergillosis with itraconazole: improvement of therapeutic efficacy by early diagnosis: Therapie 
der invasiven Aspergillose mit Itraconazol: Verbesserung des Therapieeffektes durch Frühdiagnose. Mycoses 2009, 34, 385-394.

(8) McGinnis, M. R.; Nordoff, N.; Li, R. K.; Pasarell, L.; Warnock, D. W. Sporothrix schenckii sensitivity to voriconazole, itraconazole and amphotericin B. Med. Mycol. 2001, 39, 369-371.

(9) Graybill, J. R.; Stevens, D. A.; Galgiani, J. N.; Dismukes, W. E.; Cloud, G. A. Itraconazole treatment of coccidioidomycosis. NAIAD Mycoses Study Group. Am. J. Med. 1990, 89, 282-290.

(10) Tucker, R. M. Itraconazole Therapy for Chronic Coccidioidal Meningitis. Ann. Intern. Med. 1990, 112, 108.

(11) Dismukes, W. E.; Bradsher, R. W.; Cloud, G. C.; Kauffman, C. A.; Chapman, S. W.; George, R. B.; Stevens, D. A.; Girard, W. M.; Saag, M. S.; Bowles-Patton, C. Itraconazole therapy for blastomycosis and histoplasmosis. Am. J. Med. 1992, 93, 489-497.

(12) Caillot, D.; Bassaris, H.; McGeer, A.; Arthur, C.; Prentice, H. G.; Seifert, W.; De Beule, K. Intravenous itraconazole followed by oral itraconazole in the treatment of invasive pulmonary aspergillosis in patients with hematologic malignancies, chronic granulomatous disease, or AIDS. Clin. Infect. Dis. 2001, 33, e83-90.

(13) Rawlinson, W. D.; Packham, D. R.; Gardner, F. J.; MacLEOD, C. Histoplasmosis in the acquired immunodeficiency syndrome (AIDS). Aust. NZ J. Med. 1989, 19, 707-709.

(14) Galgiani, J. N. et al. Executive Summary: 2016 Infectious Diseases Society of America (IDSA) Clinical Practice Guideline for the Treatment of Coccidioidomycosis. Clin. Infect. Dis. 2016, 63, 717-722.

(15) Chapman, S. W.; Dismukes, W. E.; Proia, L. A.; Bradsher, R. W.; Pappas, P. G.; Threlkeld, M. G.; Kauffman, C. A.; Infectious Diseases Society of America, Clinical 
practice guidelines for the management of blastomycosis: 2008 update by the Infectious Diseases Society of America. Clin. Infect. Dis. 2008, 46, 1801-1812.

(16) Supparatpinyo, K.; Chiewchanvit, S.; Hirunsri, P.; Baosoung, V.; Uthammachai, C.; Chaimongkol, B.; Sirisanthana, T. An efficacy study of itraconazole in the treatment of Penicillium marneffei infection. J. Med. Assoc. Thai. 1992, 75, 688-691.

(17) Schloer, S.; Goretzko, J.; Kühnl, A.; Brunotte, L.; Ludwig, S.; Rescher, U. The clinically licensed antifungal drug itraconazole inhibits influenza virus in vitro and in vivo. Emerg. Microbes Infect. 2019, 8, 80-93.

(18) Peyton, L.; Gallagher, S.; Hashemzadeh, M. Triazole Antifungals: A Review. Drug Today 2015, 51, 705-718.

(19) Strushkevich, N.; Usanov, S. A.; Park, H.-W. Structural basis of human CYP51 inhibition by antifungal azoles. J. Mol. Biol. 2010, 397, 1067-1078.

(20) Falci, D. R.; Pasqualotto, A. C. Profile of isavuconazole and its potential in the treatment of severe invasive fungal infections. Infect. Drug Resist. 2013, 6, 163-174.

(21) Rozman, D.; Strömstedt, M.; Tsui, L.-C.; W.Scherer, S.; R.Waterman, M. Structure and Mapping of the Human Lanosterol $14 \alpha$ - Demethylase Gene (CYP51) Encoding the Cytochrome P450 Involved in Cholesterol Biosynthesis; Comparison of Exon/Intron Organization with other Mammalian and Fungal CYP Genes. Genomics 1996, 38, 371381.

(22) Willems, L.; Geest, R. V. D.; Beule, K. D. Itraconazole oral solution and intravenous formulations: a review of pharmacokinetics and pharmacodynamics. J. Clin. Pharm. Ther. 2001, 26, 159-169.

(23) Peeters, J.; Neeskens, P.; Tollenaere, J. P.; Van Remoortere, P.; Brewster, M. E. Char- 
acterization of the interaction of 2-hydroxypropyl-beta-cyclodextrin with itraconazole at $\mathrm{pH}$ 2, 4, and 7. J. Pharm. Sci. 2002, 91, 1414-1422.

(24) Rhee, Y.-S.; Park, C.-W.; Nam, T.-Y.; Shin, Y.-S.; Chi, S.-C.; Park, E.-S. Formulation of parenteral microemulsion containing itraconazole. Arch. Pharm. Res. 2007, 30, 114123.

(25) Gajra, B.; Dalwadi, C.; Patel, R. Formulation and optimization of itraconazole polymeric lipid hybrid nanoparticles (Lipomer) using box behnken design. DARU J. Pharm. Sci. 2015, 23.

(26) Sercombe, L.; Veerati, T.; Moheimani, F.; Wu, S. Y.; Sood, A. K.; Hua, S. Advances and Challenges of Liposome Assisted Drug Delivery. Front. Pharmacol. 2015, 6, 1-13.

(27) Moghimi, S. M.; Szebeni, J. Stealth liposomes and long circulating nanoparticles: critical issues in pharmacokinetics, opsonization and protein-binding properties. Prog. Lipid Res. 2003, 42, 463-478.

(28) Romberg, B.; Oussoren, C.; Snel, C. J.; Carstens, M. G.; Hennink, W. E.; Storm, G. Pharmacokinetics of poly(hydroxyethyl-l-asparagine)-coated liposomes is superior over that of PEG-coated liposomes at low lipid dose and upon repeated administration. BBA - Biomembranes 2007, 1768, 737-743.

(29) Curić, A.; Reul, R.; Möschwitzer, J.; Fricker, G. Formulation optimization of itraconazole loaded PEGylated liposomes for parenteral administration by using design of experiments. Int. J. Pharm. 2013, 448, 189-197.

(30) Le Conte, P.; Joly, V.; Saint-Julien, L.; Gillardin, J.-M.; Carbon, C.; Yeni, P. Tissue Distribution and Antifungal Effect of Liposomal Itraconazole in Experimental Cryptococcosis and Pulmonary Aspergillosis. Am. Rev. Respir. Dis. 1992, 145, 424-429. 
(31) Wang, J.; Huang, G. Preparation of itraconazole-loaded liposomes coated by carboxymethyl chitosan and its pharmacokinetics and tissue distribution. Drug Deliv. 2011, 18, 631-638.

(32) Dzieciuch-Rojek, M.; Poojari, C.; Bednar, J.; Bunker, A.; Kozik, B.; Nowakowska, M.; Vattulainen, I.; Wydro, P.; Kepczynski, M.; Róg, T. Effects of Membrane PEGylation on Entry and Location of Antifungal Drug Itraconazole and Their Pharmacological Implications. Mol. Pharm. 2017, 14, 1057-1070.

(33) Poojari, C.; Zak, A.; Dzieciuch-Rojek, M.; Bunker, A.; Kepczynski, M.; Róg, T. Cholesterol Reduces Partitioning of Antifungal Drug Itraconazole into Lipid Bilayers. J. Phys. Chem. B 2020, 124, 2139-2148.

(34) Poojari, C.; Wilkosz, N.; Lira, R. B.; Dimova, R.; Jurkiewicz, P.; Petka, R.; Kepczynski, M.; Róg, T. Behavior of the DPH fluorescence probe in membranes perturbed by drugs. Chem. Phys. Lipids 2019, 223, 104784.

(35) Lee, J. H.; Yeo, Y. Controlled Drug Release from Pharmaceutical Nanocarriers. Chem. Eng. Sci. 2015, 125, 75-84.

(36) Son, G.-H.; Lee, B.-J.; Cho, C.-W. Mechanisms of drug release from advanced drug formulations such as polymeric-based drug-delivery systems and lipid nanoparticles. $J$. Pharm. Investig. 2017, 47, 287-296.

(37) Hub, J. S.; Awasthi, N. Probing a continuous polar defect: A reaction coordinate for pore formation in lipid membranes. J. Chem. Theory Comput. 2017, 13, 2352-2366.

(38) Awasthi, N.; Hub, J. S. Simulations of pore formation in lipid membranes: reaction coordinates, convergence, hysteresis, and finite-size effects. J. Chem. Theory Comput. 2016, 12, 3261-3269. 
(39) Awasthi, N.; Hub, J. S. Biomembrane Simulations. Computational Studies of Biological Membranes; CRC Press, Taylor \& Francis Group, 2019.

(40) Ting, C. L.; Awasthi, N.; Müller, M.; Hub, J. S. Metastable Prepores in Tension-Free Lipid Bilayers. Phys. Rev. Lett. 2018, 120.

(41) Abidor, I.; Arakelyan, V.; Chernomordik, L.; Chizmadzhev, Y. A.; Pastushenko, V.; Tarasevich, M. Electric breakdown of bilayer lipid membranes I. The main experimental facts and their qualitative discussion. Bioelectrochem. Bioenerg. 1979, 6, 37-51.

(42) Glaser, R. W.; Leikin, S. L.; Chernomordik, L. V.; Pastushenko, V. F.; Sokirko, A. I. Reversible electrical breakdown of lipid bilayers: formation and evolution of pores. $B B A$ - Biomembranes 1988, 940, 275-287.

(43) Awasthi, N.; Kopec, W.; Wilkosz, N.; Jamróz, D.; Hub, J. S.; Zatorska, M.; Petka, R.; Nowakowska, M.; Kepczynski, M. Molecular Mechanism of Polycation-Induced Pore Formation in Biomembranes. ACS Biomater. Sci. Eng. 2019, 5, 780-794.

(44) Gordeliy, V.; Kiselev, M.; Lesieur, P.; Pole, A.; Teixeira, J. Lipid Membrane Structure and Interactions in Dimethyl Sulfoxide/Water Mixtures. Biophys. J. 1998, 75, 23432351.

(45) Yu, Z.-W.; Quinn, P. J. The modulation of membrane structure and stability by dimethyl sulphoxide (Review). Mol. Membr. Biol. 1998, 15, 59-68.

(46) Kiselev, M.; Lesieur, P.; Kisselev, A.; Grabielle-Madelmond, C.; Ollivon, M. DMSOinduced dehydration of DPPC membranes studied by X-ray diffraction, small-angle neutron scattering, and calorimetry. J. Alloys Compd. 1999, 286, 195-202.

(47) Yamashita, Y.; Kinoshita, K.; Yamazaki, M. Low concentration of DMSO stabilizes the bilayer gel phase rather than the interdigitated gel phase in dihexadecylphosphatidylcholine membrane. BBA - Biomembranes 2000, 1467, 395-405. 
(48) Bunch, W. H. The effect of DMSO on the permeation of non-electrolytes through the barnacle cell membrane. J. Cell. Physiol. 1968, 72, 49-54.

(49) He, F.; Liu, W.; Zheng, S.; Zhou, L.; Ye, B.; Qi, Z. Ion transport through dimethyl sulfoxide (DMSO) induced transient water pores in cell membranes. Mol. Membr. Biol. 2012, 29, 107-113.

(50) de Ménorval, M.-A.; Mir, L. M.; Fernández, M. L.; Reigada, R. Effects of Dimethyl Sulfoxide in Cholesterol-Containing Lipid Membranes: A Comparative Study of Experiments In Silico and with Cells. PLoS ONE 2012, 7, e41733.

(51) Smondyrev, A. M.; Berkowitz, M. L. Molecular Dynamics Simulation of DPPC Bilayer in DMSO. Biophys. J. 1999, 76, 2472-2478.

(52) Sum, A. K.; de Pablo, J. J. Molecular Simulation Study on the Influence of Dimethylsulfoxide on the Structure of Phospholipid Bilayers. Biophys. J. 2003, 85, 3636-3645.

(53) Notman, R.; den Otter, W. K.; Noro, M. G.; Briels, W.; Anwar, J. The Permeability Enhancing Mechanism of DMSO in Ceramide Bilayers Simulated by Molecular Dynamics. Biophys. J. 2007, 93, 2056-2068.

(54) Lee, Y.; Pincus, P. A.; Hyeon, C. Effects of Dimethyl Sulfoxide on Surface Water near Phospholipid Bilayers. Biophys. J. 2016, 111, 2481-2491.

(55) Notman, R.; Noro, M.; O’Malley, B.; Anwar, J. Molecular Basis for Dimethylsulfoxide (DMSO) Action on Lipid Membranes. J. Am. Chem. Soc. 2006, 128, 13982-13983.

(56) Gurtovenko, A. A.; Anwar, J. Modulating the Structure and Properties of Cell Membranes: The Molecular Mechanism of Action of Dimethyl Sulfoxide. J. Phys. Chem. B 2007, 111, 10453-10460. 
(57) Hughes, Z. E.; Mark, A. E.; Mancera, R. L. Molecular Dynamics Simulations of the Interactions of DMSO with DPPC and DOPC Phospholipid Membranes. J. Phys. Chem. $B$ 2012, 116, 11911-11923.

(58) Lin, J.; Novak, B.; Moldovan, D. Molecular Dynamics Simulation Study of the Effect of DMSO on Structural and Permeation Properties of DMPC Lipid Bilayers. J. Phys. Chem. B 2012, 116, 1299-1308.

(59) Jorgensen, W. L.; Maxwell, D. S.; Tirado-Rives, J. Development and Testing of the OPLS All-Atom Force Field on Conformational Energetics and Properties of Organic Liquids. JACS 1996, 118, 11225-11236.

(60) Maciejewski, A.; Pasenkiewicz-Gierula, M.; Cramariuc, O.; Vattulainen, I.; Rog, T. Refined OPLS all-atom force field for saturated phosphatidylcholine bilayers at full hydration. J. Phys. Chem. B 2014, 118, 4571-4581.

(61) Kulig, W.; Pasenkiewicz-Gierula, M.; Róg, T. Topologies, structures and parameter files for lipid simulations in GROMACS with the OPLS-aa force field: DPPC, POPC, DOPC, PEPC, and cholesterol. Data in Brief 2015, 5, 333-336.

(62) Kulig, W.; Pasenkiewicz-Gierula, M.; Róg, T. Cis and trans unsaturated phosphatidylcholine bilayers: A molecular dynamics simulation study. Chem. Phys. Lipids 2016, $195,12-20$.

(63) Jorgensen, W. L.; Chandrasekhar, J.; Madura, J. D.; Impey, R. W.; Klein, M. L. Comparison of simple potential functions for simulating liquid water. J. Chem. Phys. 1983, 79, 926-935.

(64) Knight, C. J.; Hub, J. S. MemGen: A general web server for the setup of lipid membrane simulation systems. Bioinformatics 2015, 31, 2897-2899. 
(65) Bussi, G.; Donadio, D.; Parrinello, M. Canonical sampling through velocity rescaling. J. Chem. Phys. 2007, 126, 014101.

(66) Nosé, S. A molecular dynamics method for simulations in the canonical ensemble. Mol. Phys. 1984, 52, 255-268.

(67) Hoover, W. G. Canonical dynamics: Equilibrium phase-space distributions. Phys Rev A 1985, 31, 1695-1697.

(68) Parrinello, M.; Rahman, A. Polymorphic transitions in single crystals: A new molecular dynamics method. J. Appl. Phys. 1981, 52, 7182-7190.

(69) Miyamoto, S.; Kollman, P. A. SETTLE: An Analytical Version of the SHAKE and RATTLE Algorithms for Rigid Water Models. J. Comp. Chem. 1992, 13, 952-962.

(70) Hess, B.; Bekker, H.; Berendsen, H. J. C.; Fraaije, J. G. E. M. LINCS: A Linear Constraint Solver for Molecular Simulations. J. Comp. Chem. 1997, 18, 1463-1472.

(71) Darden, T.; York, D.; Pedersen, L. Particle mesh Ewald: An N. $\log (\mathrm{N})$ method for Ewald sums in large systems. J. Chem. Phys. 1993, 98, 10089-10092.

(72) Essmann, U.; Perera, L.; Berkowitz, M. L.; Darden, T.; Lee, H.; Pedersen, L. G. A smooth particle mesh ewald potential. J. Chem. Phys. 1995, 103, 8577-8592.

(73) Abraham, M. J.; Murtola, T.; Schulz, R.; Páll, S.; Smith, J. C.; Hess, B.; Lindahl, E. GROMACS: High performance molecular simulations through multi-level parallelism from laptops to supercomputers. SoftwareX 2015, 1, 19-25.

(74) Hub, J. S.; Groot, B. L. d.; Spoel, D. v. d. g_wham-A Free Weighted Histogram Analysis Implementation Including Robust Error and Autocorrelation Estimates. J. Chem. Theory Comput. 2010, 6, 3713-3720.

(75) Pastor, R.; MacKerell Jr, A. Development of the CHARMM force field for lipids. $J$. Phys. Chem. Lett. 2011, 2, 1526-1532. 
(76) Jämbeck, J. P. M.; Lyubartsev, A. P. Derivation and Systematic Validation of a Refined All-Atom Force Field for Phosphatidylcholine Lipids,. J. Phys. Chem. B 2012, 116, $3164-3179$.

(77) Berger, O.; Edholm, O.; Jähnig, F. Molecular dynamics simulations of a fluid bilayer of dipalmitoylphosphatidylcholine at full hydration, constant pressure, and constant temperature. Biophys. J. 1997, 72, 2002-2013.

(78) Hess, B. Determining the shear viscosity of model liquids from molecular simulations. J. Chem. Phys. 2002, 116, 209-217.

(79) Bennett, W. D.; Sapay, N.; Tieleman, D. P. Atomistic simulations of pore formation and closure in lipid bilayers. Biophys. J. 2014, 106, 210-219.

(80) Bennett, W. D.; Tieleman, D. P. The Importance of Membrane Defects Lessons from Simulations. Acc. Chem. Res. 2014, 47, 2244-2251.

(81) Tarek, M. Membrane electroporation: a molecular dynamics simulation. Biophys. J. 2005, 88, 4045-4053.

(82) Gurtovenko, A. A.; Vattulainen, I. Pore formation coupled to ion transport through lipid membranes as induced by transmembrane ionic charge imbalance: atomistic molecular dynamics study. J. Am. Chem. Soc. 2005, 127, 17570-17571.

(83) Böckmann, R. A.; De Groot, B. L.; Kakorin, S.; Neumann, E.; Grubmüller, H. Kinetics, statistics, and energetics of lipid membrane electroporation studied by molecular dynamics simulations. Biophys. J. 2008, 95, 1837-1850. 
Graphical TOC Entry

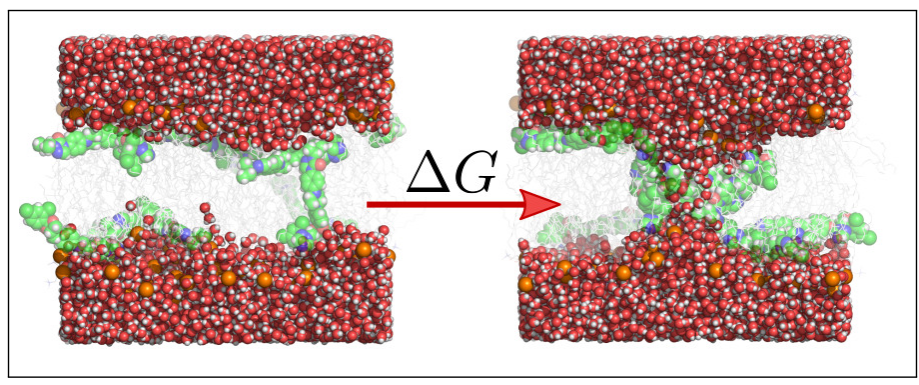

